\title{
George Graham
}

Differentiable manifolds with generalized boundary

Czechoslovak Mathematical Journal, Vol. 34 (1984), No. 1, 46-63

Persistent URL: http://dml.cz/dmlcz/101925

\section{Terms of use:}

(C) Institute of Mathematics AS CR, 1984

Institute of Mathematics of the Czech Academy of Sciences provides access to digitized documents strictly for personal use. Each copy of any part of this document must contain these Terms of use.

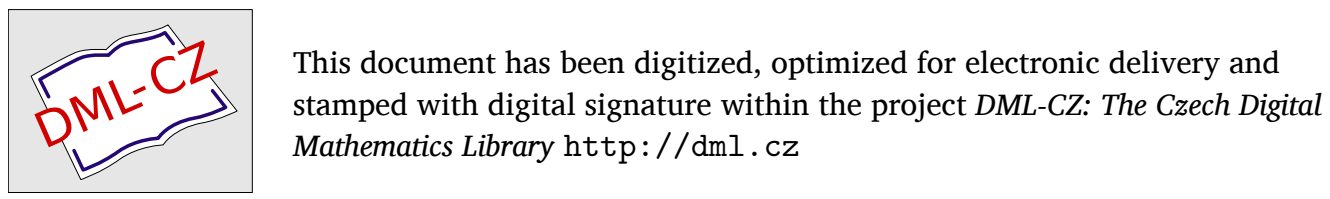




\title{
DIFFERENTIABLE MANIFOLDS WITH GENERALIZED BOUNDARY
}

\author{
George Graham ${ }^{1}$, College Station
}

(Received April 13, 1982)

1. Introduction. The purpose of this paper is to give an intrinsic description of a broad class of differentiable manifolds with generalized boundary and to show that this class includes differentiable manifolds without boundary and finite-dimensional differentiable manifolds with smooth boundary or with corners. This is accomplished by extending the usual differential calculus to include functions on arbitrary subsets of a Banach space in a way that does not require that the functions have differentiable extensions to open sets. In addition, we include some applications to the geometric theory of semigroups.

Let $\boldsymbol{E}$ be a Banach space. An $\boldsymbol{E}$-manifold with generalized boundary, or simply an $\boldsymbol{E}$-manifold, is a topological space $M$ with the property that if $p \in M$, then there is an open set $U$ about $p$ and a homeomorphism from $U$ onto a subset $A$ of $\boldsymbol{E}$ such that $\boldsymbol{A}$ has dense interior in $\boldsymbol{E}$. Differentiability of maps between manifolds is defined in terms of the differentiability of maps $f: A \subset \boldsymbol{E} \rightarrow \boldsymbol{F}$ where $A$ has dense interior in $\boldsymbol{E}$. We show that by utilizing the strong derivative (Definition 2.1) one may obtain a differential calculus for functions on arbitrary domains. In particular, we generalize the Open, Inverse, and Implicit Mapping Theorems under the assumption of strong differentiability at a point. Moreover, we show that a $k$-times strongly differentiable, or $C_{s}^{k}$, function is extendible to a $C^{k}$ function on an open set when the domain of the function is the intersection of an open set and a closed convex set in $R^{n}$ and that this result does not hold for general domains. It follows that the category of $C_{s}^{k}$ manifolds with generalized boundary properly includes $C^{k} n$-manifolds with smooth or empty boundary or with corners.

The concept of strong differentiability has been used in nonlinear analysis to prove generalized inverse and implicit mapping theorems (cf. Leach [12], Nashed [14, 15], Nijenhuis [16].) An advantage of strong differentiability is that strong differentiability at a point usually provides sufficient smoothness for the local analysis of a function via its derivative. We show that a fairly satisfactory local analysis (Theorems 2.7,

\footnotetext{
1) Portions of this paper are contained in the author's doctoral dissertation presented to the Faculty of the University of Houston in partial fulfillment of the requirements for the degree Ph.D.
} 
$2.8,2.9,2.12)$ may be made even if the point of strong differentiability is not an interior point of the domain of the function.

Differentiable manifolds with generalized boundary are of interest to us because of their importance in the development of a geometric theory of semigroups analogous to the geometric theory of Lie groups. Specifically, a $C_{s}^{k}$ semigroup is a semigroup $S$ on a $C_{s}^{k}$ manifold with generalized boundary such that the multiplication $m: S \times$ $\times S \rightarrow S$ is a $C_{s}^{k}$ map. A $C_{s}^{k}$ monoid is a $C_{s}^{k}$ semigroup with a two-sided identity element. Examples of $C_{s}^{\infty}$ monoids include Lie groups, the semigroup of all $n \times n$ real matrices, cones in $R^{n}$, and the noncommutative analogues of cones, ray semigroups (cf. $\S 5$ ). Thus by introducing the concept of a differentiable manifold with generalized boundary, we are able to give a uniform treatment to a wide variety of semigroups. For instance, we show that a $C_{s}^{k}$ monoid $(k \geqq 1)$ has cancellation in a neighborhood of its identity (Theorem 5.2). A more thorough development of the Lie-theoretic properties of $C_{s}^{k}$ semigroups is contained in [5].

The organization of the paper is as follows: $\S 2$ contains the essentials of a strong differential calculus, including generalizations of the open, inverse, and implicit mapping theorems. In $\S 3$ we establish sufficient conditions on the domain $A \subset \boldsymbol{R}^{p}$ to guarantee that each $C_{s}^{k}$ function $f: A \rightarrow \boldsymbol{R}^{q}$ is $C^{k}$ differentiable and show that this need not be the case for general domains. In $\S 4$ we define a $C_{s}^{k}$ differentiable structure and show that the category of $C_{s}^{k}$ manifolds with generalized boundary includes $C^{k}$ manifolds without boundary and finite-dimensional $C^{k}$ manifolds with smooth boundary or with corners. Applications to the theory of differentiable semigroups are contained in $\S 5$.

2. The strong derivative. Throughout this paper $\boldsymbol{E}, \boldsymbol{F}$, and $\boldsymbol{G}$ denote Banach spaces, $L(\boldsymbol{E}, \boldsymbol{F})$ denotes the Banach space of continuous linear maps from $\boldsymbol{E}$ to $\boldsymbol{F}$, and $L_{k}(\boldsymbol{E}, \boldsymbol{F})$ denotes the Banach space of continuous $k$-multilinear maps from (the product of $k$ copies of) $\boldsymbol{E}$ to $\boldsymbol{F}$. For $a \in \boldsymbol{E}$ and $\delta>0, B_{\delta}(a)$ denotes the open ball of radius $\delta$ with center $a$. We shall on occasion refer to Dieudonné [3] for additional details concerning multilinear maps and Fréchet differentiability.

Let $f: A \subset \boldsymbol{E} \rightarrow \boldsymbol{F}$ be a map and let $a \in A$. A continuous linear map $T: \boldsymbol{E} \rightarrow \boldsymbol{F}$ is a Fréchet derivative of $f$ at $a$ if for each $\varepsilon>0$, there is a $\delta>0$ such that

$$
|f(x)-f(a)-T(x-a)| \leqq \varepsilon|x-a| \text { whenever } \quad x \in B_{\delta}(a) \cap A .
$$

If $a$ is an isolated point of $A$, then any $T \in L(\boldsymbol{E}, \boldsymbol{F})$ is a Fréchet derivative of $f$ at $a$. However, if $a \in A^{0}$ (the interior of $A$ ), then there is at most one such map T. If $f$ has a unique Fréchet derivative at $a$, we shall denote it by $f^{\prime}(a)$ and say that $f^{\prime}(a)$ exists.

Let $U \subset \boldsymbol{E}$ be an open set and let $f: U \rightarrow \boldsymbol{F}$ be a map. Then $f$ is continuously Fréchet differentiable, or simply $C^{1}$, if $f^{\prime}(x)$ exists for each $x \in U$ and $f^{\prime}: U \rightarrow$ $\rightarrow L(\boldsymbol{E}, \boldsymbol{F})$ is continuous. Inductively, $f$ is $k$-times continuously Fréchet differentiable, or $C^{k}$, if $f$ is $C^{1}$ and $f^{\prime}$ is $C^{k-1}$. It follows that each $C^{k}$ map is $C^{j}$ differentiable for each $j \leqq k$, and in this case the $j^{\text {th }}$ derivative of $f$ is the continuous map $f^{(J)}=\left(f^{(j-1)}\right)^{\prime}$ 
from $U$ into the symmetric multilinear maps in $L_{j}(\boldsymbol{E}, \boldsymbol{F})$ (cf. [3, p. 176].) Finally, $f$ is infinitely Fréchet differentiable, or $C^{\infty}$, if $f$ is $C^{k}$ for each positive integer $k$.

Differentiability of a function on an arbitrary subset of $\boldsymbol{E}$ is usually defined in terms of the existence of differentiable local extensions of the function. Precisely, let $f: A \subset$ $\subset \boldsymbol{E} \rightarrow \boldsymbol{F}$. Then $f$ is $C^{k}$ differentiable if for each $a \in A$, there is an open set $U$ about $a$ and a $C^{k}$ map $g: U \rightarrow F$ such that $g$ agrees with $f$ on $U \cap A$. Defining $C^{k}$ differentiability in this way allows one to extend the usual differential calculus in a fairly straightforward manner. However, for any given function the existence of such local extensions may be difficult to verify. Moreover, such an approach is unnecessary in that it is possible to extend the differential calculus by strengthening the definition of the derivative at a point.

Let $f: A \subset \boldsymbol{E} \rightarrow \boldsymbol{F}$ be a map and let $a \in A$. A linear map $T \in L(\boldsymbol{E}, \boldsymbol{F})$ is a strong derivative of $f$ at $a$ if for each $\varepsilon>0$, there is a $\delta>0$ such that

$$
|f(y)-f(x)-T(y-x)| \leqq \varepsilon|y-x| \text { whenever } \quad x, y \in B_{\delta}(a) \cap A .
$$

It is straightforward to verify that $T$ is unique when $a \in\left(A^{0}\right)^{*}(*$ denotes closure.) If $f$ has a unique strong derivative at $a$, we shall denote it by $\mathrm{d} f(a)$ and say that $\mathrm{d} f(a)$ exists.

A subset $A$ of a topological space $X$ is admissible if $A$ has dense interior in $X$, i.e. $A \subset\left(A^{0}\right)^{*}$. Thus if $A \subset \boldsymbol{E}$ is admissible and $f: A \rightarrow \boldsymbol{F}$ is strongly differentiable at each point of $A$, then $\mathrm{d} f$ is a well-defined map from $A$ into $L(E, F)$.

Lemma 2.2. Let $A \subset B \subset X$ where $X$ is a topological space, $B$ is an admissible subset of $X$, and $A$ is an admissible subset of $B$. Then $A$ is an admissible subset of $X$. In particular, each relatively open subset of $B$ is an admissible subset of $X$.

Proof. Let $W$ be an open subset of $X$ such that $W \cap B$ is the interior of $A$ relative to $B$. Let $a \in A$ and let $U$ be an open subset of $X$ about $a$. Then $U \cap B \cap W \neq \emptyset$ since $A$ is an admissible subset of $B$. Moreover, $V=U \cap W \cap B^{0} \neq \emptyset$ by the admissibility of $B$ in $X$. But $W \cap B^{0} \subset W \cap B \subset A$ and therefore $V \subset A^{0}$. Thus $U \cap$ $\cap A^{0} \supset V \neq \emptyset$ and $A$ is an admissible set.

Each open subset of $B$ is an admissible subset of $B$ and hence an admissible subset of $X$. This completes the proof.

Although we are primarily interested in functions on admissible sets, we consider the special case when the domain is an open set. Let $U \subset \boldsymbol{E}$ be an open set and let $f: U \rightarrow \boldsymbol{F}$ be a map. If $f$ is a $C^{1}$ map, then the Fréchet derivative $f^{\prime}(a)$ is a strong derivative of $f$ at each $a \in U$, since by the mean value theorem (cf. [3, p. 156])

$$
\left|f(y)-f(x)-f^{\prime}(a)(y-x)\right| \leqq \sup _{c}\left|f^{\prime}(c)-f^{\prime}(a)\right||y-x|
$$

whenever the segment $S$ from $x$ to $y$ is contained in $U$ and the sup is taken over all $c$ in $S$. Continuity of $f^{\prime}$ at $a$ and the local convexity of $U$ yield the result that $f^{\prime}(a)$ is a strong derivative of $f$ at each point $a$ of $U$. The converse is also true as the next lemma shows. 
Lemma 2.3. Let $A \subset \boldsymbol{E}$ be an admissible set and let $f: A \rightarrow \boldsymbol{F}$ be a map. If $\mathrm{d} f(x)$ exists for each $x \in A$, then $\mathrm{d} f: A \rightarrow L(\boldsymbol{E}, \boldsymbol{F})$ is continuous.

Proof. Let $a \in A$ and let $\varepsilon>0$. Then there is a $\delta>0$ such that (2.1) holds. Let $x \in B_{\delta}(a) \cap A^{0}$ and let $v \in E$ with $|v|=1$. Since $\mathrm{d} f(x)$ exists, there is a $t>0$ such that $y=x+t v \in B_{\delta}(a) \cap A$ and

$$
|f(y)-f(x)-\mathrm{d} f(x)(y-x)| \leqq \varepsilon|y-x| .
$$

Then $|\mathrm{d} f(a) v-\mathrm{d} f(x) v|=|y-x|^{-1}|\mathrm{~d} f(a)(y-x)-\mathrm{d} f(x)(y-x)| \leqq 2 \varepsilon$. Thus $|\mathrm{d} f(a)-\mathrm{d} f(x)| \leqq 2 \varepsilon$ for each $x \in B_{\delta}(a) \cap A^{0}$.

Now let $y \in B_{\delta}(a) \cap A$. As in the preceding paragraph, there is a $\varrho>0$ such that $|\mathrm{d} f(y)-\mathrm{d} f(z)| \leqq 2 \varepsilon$ whenever $z \in B_{\varrho}(y) \cap A^{0}$. Since $A$ has dense interior, there is a $z \in B_{\delta}(a) \cap B_{\varrho}(y) \cap A^{0}$. Then $|\mathrm{d} f(y)-\mathrm{d} f(a)| \leqq 4 \varepsilon$ for each $y \in B_{\delta}(a) \cap A$. Hence $\mathrm{d} f$ is continuous at each point $a$ of $A$ and the proof is complete.

Since a strong derivative is a Fréchet derivative, we have the result that on open sets, $C^{1}$ differentiability is equivalent to strong differentiability (at each point of the open set.) By defining higher order strong differentiability inductively, we may extend this result to arbitrary orders of differentiability. For uniqueness of higher order strong derivatives, we assume that the domain of the function is an admissible set.

Let $A \subset \boldsymbol{E}$ be an admissible set and let $f: A \rightarrow \boldsymbol{F}$ be a map. Then $f$ is strongly differentiable, or simply $C_{s}^{1}$, if $\mathrm{d} f(x)$ exists for each $x \in A$. The map $f$ is $k$-times strongly differentiable, or $C_{s}^{k}$, if $f$ is $C_{s}^{1}$ and $\mathrm{d} f$ is $C_{s}^{k-1}$. The $k^{\text {th }}$ derivative of $f$ is the continuous map $\mathrm{d}^{k} f=d\left(d^{k-1} f\right): A \rightarrow L_{k}(\boldsymbol{E}, \boldsymbol{F})$. Finally, $f$ is infinitely strongly differentialbe, or $C_{s}^{\infty}$, if $f$ is $C_{s}^{k}$ for each positive integer $k$.

We have seen that $C^{1}$ differentiability is equivalent to $C_{s}^{1}$ differentiability on open sets. It follows by induction that $C^{k}$ differentiability is equivalent to $C_{s}^{k}$ differentiability on open sets, for all $k$. If $A \subset \boldsymbol{E}$ is admissible and $f: A \rightarrow \boldsymbol{F}$ is $C_{s}^{k}$ differentiable, then $f \mid A^{0}$ (the restriction of $f$ to $A^{0}$ ) is $C^{k}$ differentiable and hence $\mathrm{d}^{j} f(x)=f^{(j)}(x)$ for each $x \in A^{0}$ and each $j \leqq k$. Since $f^{(j)}(x)$ is a symmetric multilinear map when $x \in A^{0}$, it follows from the continuity of $\mathrm{d}^{j} f$ that $\mathrm{d}^{j} f(x)$ is symmetric for each $x \in A$. It also follows that a $C^{k}$ function on $A$ is $C_{s}^{k}$ differentiable, although the converse does not hold in general (cf. $\S 3$.) Hence the usual rules of computing derivatives (e.g. derivatives of constant, linear, and multilinear maps) hold.

Strong differentiability of a function at a point is a stringent but powerful condition. As Nashed [15, p. 228] observes, "strong differentiability itself is a form of the mean value theorem." It is not surprising then that strong differentiability of a function at a point implies that the function is well-behaved in a neighborhood of the point. For instance, suppose that $T \in L(\boldsymbol{E}, \boldsymbol{F})$ is a strong derivative of $f: A \subset \boldsymbol{E} \rightarrow \boldsymbol{F}$ at $a \in A$. Let $\varepsilon>0$ and $\delta>0$ be as in (2.1). Then

$$
|f(y)-f(x)-T(y-x)| \leqq \varepsilon|y-x|, \quad \text { and } \quad|f(y)-f(x)| \leqq(|T|+\varepsilon)|y-x| .
$$

Thus $f$ is Lipschitz continuous on a neighborhood of $a$. 
Theorem 2.4 (Chain Rule). Let $f: A \subset \boldsymbol{E} \rightarrow B \subset \boldsymbol{F}$ and $g: B \rightarrow \boldsymbol{G}$ be maps and let $a \in A$. If $T$ is a strong derivative of $f$ at $a$ and $S$ is a strong derivative of $g$ at $f(a)$, then $S \circ T$ is a strong derivative of $g \circ f$ at $a$. If $A$ and $B$ are admissible sets and $f$ and $g$ are $C_{s}^{k}$ differentiable, then $g \circ f$ is $C_{s}^{k}$ differentiable and

$$
\mathrm{d}(g \circ f)(x)=\mathrm{d} g(f(x)) \circ \mathrm{d} f(x)
$$

for each $x \in A$.

Proof. The proof of the first statement is a straightforward computation using the Lipschitz continuity of $f$ near $a$. The second statement follows by induction in the usual way. We remark that the proof of the second statement uses the fact that a map $h: A \rightarrow E_{1} \times \ldots \times \boldsymbol{E}_{n}$ is $C_{s}^{k}$ differentiable if and only if each component function $h_{i}: A \rightarrow \boldsymbol{E}_{i}(i=1, \ldots, n)$ is $C_{s}^{k}$ differentiable, and in this case $(\mathrm{d} h(x))_{i}=\mathrm{d}\left(h_{i}\right)(x)$.

Pointwise strong differentiability has already been used to advantage in proving generalized inverse and implicit map theorems (cf. Leach [12], Nashed [15], Nijenhuis [16].) We now extend these results to functions on arbitrary subsets of $\boldsymbol{E}$, with particular attention to the case when the domain is an admissible set. The Open Mapping Theorem will be an immediate consequence of the following theorem of Graves. We preceed the theorem with a lemma due to Banach.

Lemma 2.5. (Banach [1, p. 38]). If $T \in L(\boldsymbol{E}, \boldsymbol{F})$ maps $\boldsymbol{E}$ onto $\boldsymbol{F}$, then there is a number $M$ such that for each $y \in \boldsymbol{F}$ there is an $x \in \boldsymbol{E}$ with $T x=y$ and $|x| \leqq M|y|$.

Theorem 2.6 (Graves [6, Theorem 1]). Let $U \subset \boldsymbol{E}$ be an open set about $x_{0}$ and let $f: U \rightarrow \boldsymbol{F}$ a map. Suppose that there is a $T \in L(\boldsymbol{E}, \boldsymbol{F})$ from $\boldsymbol{E}$ onto $\boldsymbol{F}$ and positive numbers $\varepsilon$ and $\delta$ such that $B_{\delta}\left(x_{0}\right) \subset U$ and

$$
|f(y)-f(x)-T(y-x)| \leqq \varepsilon|y-x|
$$

whenever $x$ and $y$ are in $B_{\delta}\left(x_{0}\right)$, where $\varepsilon M<1$ and $M$ is a constant as in Lemma 2.5 . Then the equation $f(x)=z$ has at least one solution with $x \in B_{\delta}\left(x_{0}\right)$ whenever $z \in B_{\varrho}\left(f\left(x_{0}\right)\right)$, where $\varrho=\delta(1-M \varepsilon) M^{-1}$.

Theorem 2.7 (Open Mapping Theorem). Let $f: A \subset \boldsymbol{E} \rightarrow \boldsymbol{F}$ be a map and let $a \in A$. Suppose that $T$ is a strong derivative of $f$ at a and that $T$ maps $\boldsymbol{E}$ onto $\boldsymbol{F}$. Then there is a $\delta>0$ such that if $U=B_{\delta}(a) \cap A$, then $f \mid U^{0}$ is an open map. Moreover, $f(B)$ is an admissible subset of $\boldsymbol{F}$ whenever $B$ is an admissible subset of $\boldsymbol{E}$ contained in $U$. In particular if $A$ is admissible, then $U^{0} \neq \emptyset$ and $f(U)$ is an admissible subset of $\boldsymbol{F}$.

Proof. Let $M$ be a constant for $T$ as provided by Lemma 2.5. Pick $\varepsilon$ so that $0<$ $<\varepsilon<M^{-1}$ and let $\delta$ be as in (2.1). If $V$ is an open subset of $\boldsymbol{E}$ contained in $U=$ $=B_{\delta}(a) \cap A$ and $x_{0} \in V$, then there is a $\gamma>0$ such that $B_{\gamma}\left(x_{0}\right) \subset V$ and by Theorem 2.6, $B_{\varrho}\left(f\left(x_{0}\right)\right) \subset f\left(B_{\gamma}\left(x_{0}\right)\right)$ for some $\varrho>0$. Hence $f(V)$ is open and $f \mid U^{0}$ is an open map. 
Now let $B$ be an admissible subset of $\boldsymbol{E}$ contained in $U$. Then $f\left(B^{0}\right)$ is open in $\boldsymbol{F}$, and by the continuity of $f$ on $U, f\left(B^{0}\right)$ is dense in $f(B)$. Thus $f(B)$ is an admissible subset of $\boldsymbol{F}$. Finally, if $A$ is admissible, then $U$ is admissible by Lemma 2.2. Hence $U^{0} \neq \emptyset$ and $f(U)$ is admissible. This completes the proof.

If $f: \boldsymbol{E} \rightarrow \boldsymbol{F}$ is a $C^{\infty}$ map such that $f^{\prime}(a)$ is one-to-one for some $a \in E$, it does not follow that $f$ is one-to-one on a neighborhood of $a$. For example, let $\boldsymbol{E}$ and $\boldsymbol{F}$ be the Banach spaces $L^{4}[0,1]$ and $L^{2}[0,1]$, respectively, and let $f: \boldsymbol{E} \rightarrow \boldsymbol{F}$ be the map $f(x)=x^{2}$, i.e. $f(x)(t)=[x(t)]^{2}$ for $t \in[0,1]$. Then $f$ is $C^{\infty}$ and $f^{\prime}(x) u=2 x u$ for each $x, u \in \boldsymbol{E}$. For each $s \in[0,1]$ let $y_{s} \in L^{4}[0,1]$ be the map defined by $y_{s}(t)=t^{2}$ if $t \in(s, 1]$ and $y_{s}(t)=-t^{2}$ it $t \in[0, s]$. If $a=y_{0}$, then $f^{\prime}(a)$ is one-to-one. However, $\left\|y_{s}-a\right\|_{4}=\left(2 s^{3} / 3\right)^{1 / 4}$ and $f\left(y_{s}\right)=f(a)$. Hence $f$ is not one-to-one on any neighborhood of $a$. The problem here is that the range of $f^{\prime}(a)$ is not closed in the Hilbert space $L^{2}[0,1]$.

A continuous left inverse of a map $T \in L(\boldsymbol{E}, \boldsymbol{F})$ is a map $S \in L(\boldsymbol{F}, \boldsymbol{E})$ such that $S \circ T=1_{E}$, the identity on $\boldsymbol{E}$. It is well known that $T$ has a continuous left inverse if and only if $T$ is one-to-one and the range $R(T)$ of $T$ is closed and complemented in $\boldsymbol{F}$. Of course, if $\boldsymbol{F}$ is a Hilbert space then $T$ has a continuous left inverse if and only if $T$ is one-to-one and $R(T)$ is closed, since each closed subspace of $\boldsymbol{F}$ is complemented. Similarly, if $\boldsymbol{F}$ is finite dimensional, then $T$ has a continuous left inverse if and only if $T$ is one-to-one.

Theorem 2.8. Let $f: A \subset \boldsymbol{E} \rightarrow \boldsymbol{F}$ be a map and let $a \in A$. Suppose that $T$ is a strong derivative of $f$ at $a$ and that $T$ has a continuous left inverse $S$. Let $N$ denote the null space of $S$. Then:

(i) there is a $\delta>0$ such that $f \mid U$ is continuous and

$$
|y-x| \leqq 2|S f(y)-S f(x)| \leqq 2|S| \cdot|f(y)-f(x)|
$$

whenever $x, y \in U$, where $U=B_{\delta}(a) \cap A$. Hence $f \mid U$ is one-to-one and continuous and $(f \mid U)^{-1}$ is continuous on $f(U)$.

(ii) if $\delta$ is any positive number such that $f \mid U$ is continuous and (*) holds for $x, y \in U=B_{\delta}(a) \cap A$, then there is a continuous map $g: f(U)+N \rightarrow U$ such that

$$
g(f(x))=x \text { and } g^{-1}(x)=f(x)+N, \text { for each } x \in U .
$$

Furthermore, $S$ is a strong derivative of $g$ at each point of $f(a)+N$, and in particular, $S$ is a strong derivative of $(f \mid U)^{-1}$ at $f(a)$. Finally, the set $f(U)+N$ is admissible in $\boldsymbol{F}$ if $\boldsymbol{A}$ is admissible in $\boldsymbol{E}$.

Proof. (i) Let $h(x)=S f(x)-x$ for $x \in A$. Then $0 \in L(\boldsymbol{E}, \boldsymbol{E})$ is a strong derivative of $h$ at $a$ by the chain rule. Hence there is a $\delta>0$ such that if $U=B_{\delta}(a) \cap A$, then $f$ is continuous on $U$ and

$$
|h(y)-h(x)|=|S f(y)-y-S f(x)+x| \leqq \frac{1}{2}|y-x|
$$


whenever $x, y \in U$. Thus if $x, y \in U$, then

and

$$
\begin{gathered}
|y-x| \leqq|S f(x)-x-S f(y)+y|+|S f(y)-S f(x)| \leqq \\
\leqq \frac{1}{2}|y-x|+|S f(y)-S f(x)|,
\end{gathered}
$$

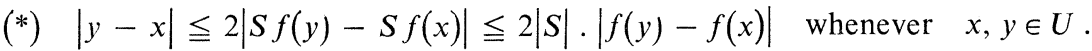

It follows that $f \mid U$ is one-to-one and that $(f \mid U)^{-1}$ is continuous on $f(U)$.

(ii) Let $\delta$ and $U$ be as in (i) and let $H=S f \mid U$. Inequality (*) implies that $H$ is one-to-one on $U$ and that $H^{-1}: H(U) \rightarrow U$ is continuous. Let $B=S^{-1}(H(U))=$ $=S^{-1}(S f(U))$ and let $g: B \rightarrow U$ be the continuous map $H^{-1} \circ S$. If $x \in U$ then

$$
g(f(x))=H^{-1}(S f(x))=x .
$$

Now $z \in B$ implies $g(z)=H^{-1}(S z)$ and $x \in U$ implies $x=H^{-1}(H(x))=$ $=H^{-1}(S f(x))$. Thus $g(z)=x$ if and only if $S z=S f(x)$, i.e. if and only if $z \in$ $\in f(x)+N$. Hence

$$
g^{-1}(x)=f(x)+N, \text { for each } x \in U .
$$

It follows that $B=f(U)+N$ and that each $z \in B$ can be written uniquely in the form $z=f(x)+v$ with $x \in U$ and $v \in N$, namely $x=g(z)$ and $v=z-f(g(z))$.

Fix $u \in N$ and let $\bar{a}=f(a)+u$. We show now that $S$ is a strong derivative of $g$ at $\bar{a}$. We assume that $|S| \neq 0$ since $\boldsymbol{E}$ is trivial if $|S|=0$. Let $\varepsilon>0$. Then there is a positive number $\eta<\delta$ such that

$$
|f(y)-f(x)-T(y-x)| \leqq \frac{1}{2} \varepsilon|S|^{-2}|y-x| \text { whenever } \quad x, y \in B_{\eta}(a) \cap A .
$$

Now let $\varrho=\frac{1}{2} \eta|S|^{-1}$ and let $\bar{x}, \bar{y} \in B_{\varrho}(\bar{a}) \cap B$. Then $\bar{x}=f(x)+v$ and $\bar{y}=f(y)+w$ for some $x, y \in U$ and $v, w \in N$. It follows that $S \bar{x}=S f(x), S \bar{y}=S f(y), g(\bar{x})=x$, and $g(\bar{y})=y$. By $(*)$,

$$
|y-a| \leqq 2|S f(y)-S f(a)|=2|S \bar{y}-S \bar{x}| \leqq 2|S| \cdot|\bar{y}-\bar{x}|<\eta,
$$

and similarly for $x$. Thus

$$
\begin{aligned}
& |g(\bar{y})-g(\bar{x})-S(\bar{y}-\bar{x})|=|y-x-S(f(y)-f(x))|= \\
& =|S(T(y-x)-f(y)+f(x))| \leqq|S| \frac{1}{2} \varepsilon|S|^{-2}|y-x| \leqq \\
& \leqq \varepsilon|S|^{-1}|S f(y)-S f(x)|=\varepsilon|S|^{-1}|S \bar{y}-S \bar{x}| \leqq \varepsilon|\bar{y}-\bar{x}| .
\end{aligned}
$$

Hence $S$ is a strong derivative of $g$ at each point of $f(a)+N$.

Finally, suppose that $A$ is admissible. Then $U$ is admissible by Lemma 2.2. The inequality

$$
|H(y)-H(x)-(y-x)|=|S f(y)-y-S f(x)+x| \leqq \frac{1}{2}|y-x|
$$

and Theorem 2.6 imply that $H\left(U^{0}\right) \subset H(U)^{0}$. Then $g^{-1}\left(U^{0}\right)=S^{-1}\left(H\left(U^{0}\right)\right)$ is open in $\boldsymbol{F}$ and contained in $B$. If $z \in B$ then there is a net $\left\{x_{\alpha}\right\}$ of points in $U^{0}$ converging 
to $g(z)$. Since $f$ is continuous on $U$, the net $\left\{f\left(x_{\alpha}\right)+z-f(g(z))\right\}$ converges to $z$ and lies in $B^{0}$. Thus $B$ is admissible and the proof is complete.

Theorem 2.9 (Inverse Function Theorem). Let $A \subset \boldsymbol{E}$ be an admissible set containing $a$ and let $f: A \rightarrow \boldsymbol{F}$ be a map. Suppose that $\mathrm{d} f(a)$ exists and is an isomorphism. Then there is a $\delta>0$ such that $f(U)$ is an admissible subset of $\boldsymbol{F}, f \mid U$ : $: U \rightarrow f(U)$ is a homeomorphism, and $\mathrm{d} f(a)^{-1}$ is a strong derivative of $(f \mid U)^{-1}$ at $f(a)$, where $U=B_{\delta}(a) \cap A$. Moreover, if $f$ is $C_{s}^{k}$ differentiable, then $\delta$ can be chosen so that $(f \mid U)^{-1}$ is $C_{s}^{k}$ differentiable.

Proof. The proof of the first statement is a straightforward application of Corollary 2.7 and Theorem 2.8. Suppose that $f$ is $C_{s}^{k}$ differentiable. Then $\mathrm{d} f: A \rightarrow L(\boldsymbol{E}, \boldsymbol{F})$ is continuous and $C_{s}^{k-1}$ (if $k>1$ ). Now the collection $\mathscr{H}$ of isomorphisms in $L(\boldsymbol{E}, \boldsymbol{F})$ is an open set, and the map $\theta(T)=T^{-1}$ from $\mathscr{H}$ into $L(\boldsymbol{F}, \boldsymbol{E})$ is $C^{\infty}$ (cf. [3, p. 148]) and therefore $C_{s}^{\infty}$. Thus $\delta$ may be chosen sufficiently small that $\mathrm{d} f(x) \in \mathscr{H}$ for each $x \in U=B_{\delta}(a) \cap A, f(U)$ is admissible, and $f \mid U: U \rightarrow f(U)$ is a homeomorphism. Let $g=(f \mid U)^{-1}$. Then $g$ is continuous and $\mathrm{d} g(f(x))=\mathrm{d} f(x)^{-1}$ for each $x \in U$, i.e. $\mathrm{d} g=\theta \circ \mathrm{d} f \circ g$. By induction, $g$ is $C_{s}^{k}$ differentiable.

Theorem 2.10. Let $A \subset \boldsymbol{E}$ be an admissible set containing $a$ and let $f: A \rightarrow \boldsymbol{F}$ be a $C_{s}^{k}$ map. Suppose that $\mathrm{d} f(a)$ has a continuous left inverse $S$ and let $N$ be the null space of $S$. Then there is a $\delta>0$, an admissible subset $B$ of $\boldsymbol{F}$, and a $C_{s}^{k}$ map $g: B \rightarrow A$ such that

$$
g(f(x))=x \text { and } g^{-1}(x)=f(x)+N \text { whenever } x \in B_{\delta}(a) \cap A .
$$

Proof. Since $\mathrm{d} f$ is continuous, there is a $\varrho>0$ such that $S \circ \mathrm{d} f(x)$ is an isomorphism for $x \in B_{\varrho}(a) \cap A$. By Theorem 2.8 there is a positive number $\delta<\varrho$ and a continuous map $g: f(U)+N \rightarrow U$, where $U=B_{\delta}(a) \cap A$, such that $B=f(U)+$ $+N$ is admissible and if $x \in U$, then $g(f(x))=x, g^{-1}(x)=f(x)+N$, and $S \circ \mathrm{d} f(x)$ is an isomorphism. Form the product space $\boldsymbol{E} \times \boldsymbol{N}$ and let $\pi_{i}(i=1,2)$ be projection onto the $i^{\text {th }}$ factor. Define $\phi: U \times N \rightarrow B$ by $\phi(x, v)=f(x)+v$. Then $\phi$ is continuous and $C_{s}^{k}$ differentiable.

If $z \in B$ then $z-f(g(z)) \in N$. Define $\psi: B \rightarrow U \times N$ by $\psi(z)=(g(z), \quad z-$ $-f(g(z))$. Then $\phi \circ \psi(z)=z$ for each $z \in B$, and $\psi(\phi(x, v))=\psi(f(x)+v)=(x, v)$ for each $(x, v) \in U \times N$. Since $g$ is continuous, $\psi$ is continuous, and $\phi: U \times N \rightarrow B$ is a homeomorphism with $\phi^{-1}=\psi$. Moreover, $g=\pi_{1} \circ \phi^{-1}$.

Let $x \in U$ and $v \in \boldsymbol{N}$. Then $\mathrm{d} \phi(x, v)(u, w)=\mathrm{d} f(x) u+w$ for each $(u, w) \in \boldsymbol{E} \times \boldsymbol{N}$. If $\mathrm{d} \phi(x, v)(u, w)=0$, then $S \mathrm{~d} f(x) u=0$, and hence $u=0$ and $w=0$. Thus $\mathrm{d} \phi(x, v)$ is one-to-one. If $z \in \boldsymbol{F}$, then there is a (unique) $u \in \boldsymbol{E}$ such that $S \mathrm{~d} f(x) u=$ $=S z$ and $\mathrm{d} \phi(x, v)(u, z-\mathrm{d} f(x) u)=z$, i.e. $\mathrm{d} \phi(x, v)$ maps onto $\boldsymbol{F}$. It follows that $\mathrm{d} \phi(x, v)$ is an isomorphism for each $x \in U$ and each $v \in \boldsymbol{N}$ and that $\phi^{-1}$ is $C_{s}^{k}$ differentiable. Since $g=\pi_{1} \circ \phi^{-1}, g$ is $C_{s}^{k}$ differentiable by the chain rule. 
Theorem 2.11 (Parameterized Mapping and Implicit Mapping Theorems). Let $a \in A \subset \boldsymbol{E}, b \in B \subset \boldsymbol{F}$, and $f: A \times B \rightarrow \boldsymbol{G}$. Suppose that $T$ is a strong derivative of $f$ at $(a, b)$ and that the map $T_{2}: \boldsymbol{F} \rightarrow \boldsymbol{G}$ defined by $T_{2}(v)=T(0, v)$ is an isomorphism. Then:

(i) there is a $\delta>0$ such that for $U_{1}=B_{\delta}(a) \cap A$ and $V_{1}=B_{\delta}(b) \cap B$, if $x \in U_{1}$ then the map $f_{x}: V_{1} \rightarrow \boldsymbol{G}$ defined by $f_{x}(z)=f(x, z)$ is one-to-one and both $f_{x}$ and $f_{x}^{-1}$ are Lipschitz continuous. Moreover, $f_{x}\left(V_{1}^{0}\right)$ is an open subset of $\boldsymbol{G}$ for each $x \in U_{1}$.

(ii) if $V \subset \boldsymbol{F}$ is an open set with $c \in V \subset B$, for some $c \in V_{1}$, then there is an open set $W \subset \boldsymbol{G}$ about $f(a, c)$ and an $r>0$ such that $W \subset f_{x}(V)$ for each $x \in B_{r}(a) \cap$ $\cap A$.

(iii) if $U, V$, and $W$ are sets with $U \subset U_{1}, V \subset V_{1}$, and $W \subset f_{x}(V)$ for each $x \in U$, then there is a continuous map $g: U \times W \rightarrow V$ such that

$$
f(x, g(x, w))=w \quad \text { for each } \quad(x, w) \in U \times W .
$$

Furthermore, if $A$ and $B$ are admissible sets and $f$ is $C_{s}^{k}$ differentiable, then $\delta$ can be chosen so that all of the maps $f_{x}, f_{x}^{-1}$, and $g$ are $C_{s}^{k}$ differentiable.

Proof. Let $\hat{\phi}: A \times B \rightarrow \boldsymbol{E} \times \boldsymbol{G}$ be the map $\hat{\phi}=\pi_{1} \times f$. Then $\left(\pi_{1} \times T\right)$ is a strong derivative of $\hat{\phi}$ at $(a, b)$ and is an isomorphism. By Theorem 2.8 and the definition of strong differentiability, there is a $\delta>0$ such that for $U_{1}=B_{\delta}(a) \cap A$, $V_{1}=B_{\delta}(b) \cap B$, and $N=2\left|\left(\pi_{1} \times T\right)^{-1}\right|$

$$
\begin{gathered}
|(y, w)-(x, z)| \leqq N|\hat{\phi}(y, w)-\hat{\phi}(x, z)| \text { and } \\
|\hat{\phi}(y, w)-\hat{\phi}(x, z)-(y-x, T(y-x, w-z))| \leqq N^{-1}|(y-x, w-z)| \\
\text { whenever }(x, z),(y, w) \in U_{1} \times V_{1} .
\end{gathered}
$$

Let $\phi=\hat{\phi} \mid U_{1} \times V_{1}$. Inequality (*) implies that $\phi$ is one-to-one and that $\phi^{-1}$ is continuous. Inequality $(* *)$ implies that

$$
|\phi(y, w)-\phi(x, z)| \leqq K|(y-x, w-z)| \text { for }(x, z),(y, w) \in U_{1} \times V_{1},
$$

where $K=\left|\pi_{1} \times T\right|+N^{-1}$ and hence $\phi$ is continuous.

For each $x \in U_{1}$ let $f_{x}: V_{1} \rightarrow \boldsymbol{G}$ be the map $f_{x}(z)=f(x, z)$. Then

$$
\begin{gathered}
\left|f_{x}(z)-f_{x}(w)\right|=\mid(x-x, f(x, z)-f(x, w) \mid= \\
=|\phi(x, z)-\phi(x, w)| \leqq K|z-w| \text { for } z, w \in V_{1} .
\end{gathered}
$$

Thus each map $f_{x}$ is Lipschitz continuous. In a similar manner,

$$
\begin{gathered}
|z-w| \leqq N\left|f_{x}(z)-f_{x}(w)\right| \text { and } \\
\left|f_{x}(z)-f_{x}(w)-T_{2}(z-w)\right| \leqq N^{-1}|z-w| \text { for } z, w \in V_{1} .
\end{gathered}
$$


Hence each map $f_{x}$ is one-to-one and $f_{x}^{-1}$ is Lipschitz continuous. By Theorem 2.6 (with $M=\frac{1}{2} N$ and $\varepsilon=N^{-1}$ ), $f_{x}\left(V_{1}^{0}\right)$ is open in $\boldsymbol{G}$. If $B$ is an admissible set, then $V_{1}^{0} \neq \emptyset$ and $f_{x}\left(V_{1}\right)$ is an admissible subset of $\boldsymbol{G}$ for each $x \in U_{1}$. This completes the proof of (i).

For (ii), let $V \subset \boldsymbol{F}$ be an open set with $V \subset B$ and $V \cap V_{1} \neq \emptyset$. Let $c \in V \cap V_{1}$. There is an $\eta>0$ such that $B_{\eta}(c) \subset V \cap V_{1}$. By Theorem 2.6 and inequality (**),

$$
B_{\varrho}\left(f_{x}(c)\right) \subset f_{x}\left(B_{\eta}(c)\right) \subset f_{x}(V) \text { for each } x \in U_{1},
$$

where $\varrho=\eta N^{-1}$. Let $\mu=\frac{1}{2} \varrho, W=B_{\mu}(f(a, c))$, and $r=\min \left\{\frac{1}{2} \mu K^{-1}, \delta\right\}$. If $x \in$ $\in B_{r}(a) \cap A$, then

$$
\left|f_{x}(c)-f_{a}(c)\right| \leqq|\phi(x, c)-\phi(a, c)| \leqq K|x-a|<\mu .
$$

Moreover, if $w \in W$, then $\left|w-f_{x}(c)\right| \leqq\left|w-f_{a}(c)\right|+\left|f_{a}(c)-f_{x}(c)\right|<\varrho$. Hence, $W \subset B_{\varrho}\left(f_{x}(c)\right) \subset f_{x}(V)$ for each $x \in B_{r}(a) \cap A$ and the proof of (ii) is complete.

For (iii), suppose that $U, V$, and $W$ are sets with $U \subset U_{1}, V \subset V_{1}$, and $W \subset f_{x}(V)$ for each $x \in U$. Then $U \times W \subset \phi(U \times V)$. Let $\psi=\phi^{-1} \mid U \times W$. If $(x, w) \in U \times$ $\times W$, then $(x, w)=\phi(\psi(x, w))=\phi\left(\psi_{1}(x, w), \psi_{2}(x, w)\right)=\left(\psi_{1}(x, w), f(\psi(x, w))\right)$. Thus $\psi_{1}(x, w)=x$ and $f\left(x, \psi_{2}(x, w)\right)=w$ for each $x \in U, w \in W$. Let $g$ be the continuous map $\psi_{2}$ and (iii) is proved.

Finally, suppose that $A$ and $B$ are admissible sets and that $f$ is $C_{s}^{k}$ differentiable. Then there is a $\delta>0$ such that, in addition to inequalities $\left(^{*}\right)$ and $(* *)$, the map $\mathrm{d}_{2} f(x, z): \boldsymbol{F} \rightarrow \boldsymbol{G}$ defined by $\mathrm{d}_{2} f(x, z)(v)=\mathrm{d} f(x, z)(0, v)$ is an isomorphism for each $(x, z) \in U_{1} \times V_{1}$. Then the map $\phi$ defined above is one-to-one and continuous, $\phi^{-1}$ is continuous, and $\mathrm{d} \phi(x, z)$ is an isomorphism for each $(x, z) \in U_{1} \times V_{1}$. It follows that the maps $\phi^{-1}$ and $g$ defined above are $C_{s}^{k}$ differentiable. Since each $f_{x}$ is a topological embedding and $\mathrm{d} f_{x}(z)=\mathrm{d}_{2} f(x, z)$, it also follows that $f_{x}$ and $f_{x}^{-1}$ are $C_{s}^{k}$ differentiable for each $x \in U_{1}$. This completes the proof of Theorem 2.11.

3. Extension of strongly differentiable functions. In $\S 2$ a function $f: A \subset \boldsymbol{E} \rightarrow \boldsymbol{F}$ was defined to be $C^{k}$ differentiable if and only if for each $a \in A$, there is an open set $U$ about $a$ and a $C^{k}$ map $g: U \rightarrow F$ such that $f$ and $g$ agree on $U \cap A$. Since $C^{k}$ and $C_{s}^{k}$ differentiability are equivalent on open sets, it follows that $C^{k}$ differentiability of $f$ implies $C_{s}^{k}$ differentiability of $f$ when the domain $A$ of $f$ is an admissible set. The converse does not hold in general. For example, let $A \subset \boldsymbol{R}^{2}$ be $\left\{x=\left(x_{1}, x_{2}\right): x_{1} \neq\right.$ $\neq 0\} \cup\{(0,0)\}$ and let $f: A \rightarrow \boldsymbol{R}$ be the map defined by $f(x)=f\left(x_{1}, x_{2}\right)=x_{1} x_{2}$ if $x_{2}>0$ and $f(x)=0$ otherwise. The map $f$ is strongly differentiable on $A$, but the unique continuous extension of $f$ to $\boldsymbol{R}^{2}$ is not Fréchet differentiable at points $\left(x_{1}, x_{2}\right)$ with $x_{1} \neq 0$ and $x_{2}=0$. Thus $f$ is not $C^{\mathbf{1}}$. In this section we show that a $C_{s}^{k}$ map $f: A \subset \boldsymbol{R}^{p} \rightarrow \boldsymbol{R}^{q}$ has a $C^{k}$ extension to an open set about $A$ provided $A$ is closed and either $k=1$ or $A$ is convex. This result follows from a theorem of Whitney.

Definition 3.1 (Whitney [18].) Let $f: A \subset \boldsymbol{R}^{p} \rightarrow \boldsymbol{R}^{q}$ be a map and $k$ a positive integer. For each positive integer $n \leqq k$ let $D^{n} f$ be a map from $A$ into $L_{n}^{s}\left(\boldsymbol{R}^{p}, \boldsymbol{R}^{q}\right)$, 
the space of symmetric $n$-multilinear maps from $\boldsymbol{R}^{p}$ to $\boldsymbol{R}^{q}$. Then $f$ is $C^{k}$ differentiable in the sense of Whitney, or simply $C_{w}^{k}$, in terms of $\left\{\mathrm{D}^{n} f\right\}_{n=1}^{k}$ if for each $a \in A$ and each $\varepsilon>0$, there is a $\delta>0$ such that

$$
\begin{gathered}
\left|\mathrm{D}^{m} f(y)-\sum_{n=0}^{k-m} \frac{1}{n !} \mathrm{D}^{m+n} f(x)(y-x)^{n} \leqq \varepsilon\right| y-\left.x\right|^{k-m}, \\
\text { whenever } m \leqq k \text { and } x, y \in B_{\delta}(a) \cap A .
\end{gathered}
$$

Here $\mathrm{D}^{0} f=f$ and $\mathrm{D}^{m+n} f(x)(y-x)^{n}$ is the $m$-multilinear map which results from placing $y-x$ in $n$ of the arguments of $\mathrm{D}^{m+n} f(x)$. The map $f$ is $C^{\infty}$ in the sense of Whitney, or $C_{w}^{\infty}$, in terms of $\left\{\mathrm{D}^{n} f\right\}_{n=1}^{\infty}$ if $f$ is $C_{w}^{k}$ in terms of $\left\{\mathrm{D}^{n} f\right\}_{n=1}^{k}$ for each positive integer $k$.

Let $f: A \subset \boldsymbol{R}^{p} \rightarrow \boldsymbol{R}^{q}$ be $C_{w}^{k}$ in terms of $\left\{\mathrm{D}^{n} f\right\}_{n=1}^{k}$. Then it can be shown easily that $f$ and each of the maps $\mathrm{D}^{n} f$ are continuous. It also follows that if $n, m, n+m \leqq$ $\leqq k$, then $\mathrm{D}^{n} f$ is $C_{w}^{m}$ in terms of $\left\{\mathrm{D}^{n+j} f\right\}_{j=1}^{m}$. In particular, for any $a \in A$ and any $\varepsilon>0$, there is a $\delta>0$ such that

$$
|f(y)-f(x)-\mathrm{D} f(x)(y-x)| \leqq \varepsilon|y-x| \text { whenever } \quad x, y \in B_{\delta}(a) \cap A .
$$

Thus $\mathrm{D} f$ is a locally uniform differential in the sense of Vainberg ([17].) Since $\mathrm{D} f$ is continuous and

$$
|f(y)-f(x)-\mathrm{D} f(a)(y-x)| \leqq \varepsilon|y-x|+|\mathrm{D} f(x)-\mathrm{D} f(a)| \cdot|y-x|
$$

for $x, y \in B_{\delta}(a) \cap A$, it follows that $\mathrm{D} f(a)$ is a strong derivative of $f$ at $a$. By induction, $f$ is $C_{s}^{k}$ differentiable when $f$ is $C_{w}^{k}$ differentiable in terms of $\left\{\mathrm{D}^{n} f\right\}_{n=1}^{k}$ and $A$ is admissible, and in this case $\mathrm{D}^{n} f(x)=\mathrm{d}^{n} f(x)$ for each $x \in A$ and each $n=1, \ldots, k$. A much stronger statement can be made when $A$ is closed.

Theorem 3.2 (Whitney [18, Theorem I]). Let $A \subset \boldsymbol{R}^{p}$ be a closed set and $f: A \rightarrow \boldsymbol{R}^{q}$ a map. Suppose that $f$ is $C_{w}^{k}$ in terms of $\left\{\mathrm{D}^{n} f\right\}_{n=1}^{k}$. Then there is a $C^{k}$ map $g: \boldsymbol{R}^{p} \rightarrow$ $\rightarrow \boldsymbol{R}^{q}$ such that $g \mid A=f$ and $g^{(n)}(x)=\mathrm{D}^{n} f(x)$ for each $x \in A$ and each $n=1, \ldots, k$.

Taylor's theorem yields the converse to Whitney's theorem. Specifically, if $f: \boldsymbol{R}^{p} \rightarrow$ $\rightarrow \boldsymbol{R}^{q}$ is $C^{k}$ differentiable and $A \subset \boldsymbol{R}^{p}$, then $f \mid A$ is $C_{w}^{k}$ in terms of $\left\{f^{(n)} \mid A\right\}_{n=1}^{k}$. Thus the concepts of $C^{k}$ differentiability and $C_{w}^{k}$ differentiability are equivalent on closed sets and on open sets.

We have already seen that if $A \subset \boldsymbol{R}^{p}$ is an admissible set and $f: A \rightarrow \boldsymbol{R}^{q}$ is $C_{w}^{k}$ in terms of some $\left\{\mathbf{D}^{n} f\right\}_{n=1}^{k}$, then $f$ is $C_{s}^{k}$ differentiable. A similar computation shows that if $f$ is $C_{s}^{1}$, then $f$ is $C_{w}^{1}$ in terms of $\{\mathrm{d} f\}$. Hence we have the following corollary to Whitney's theorem.

Corollary 3.3. Let $A \subset \boldsymbol{R}^{p}$ be a closed admissible set (i.e. the closure of an open set) and let $f: A \rightarrow \boldsymbol{R}^{q}$ be a map. Then these are equivalent:

(1) The mapf is $C_{s}^{\mathbf{1}}$ differentiable. 
(2) The map $f$ is $C_{w}^{1}$ differentiable in terms of some $\{\mathrm{D} f\}$.

(3) The map $f$ is $C^{1}$ differentiable.

This result does not extend to higher orders of differentiability without additional constraints on the set $A$ (e.g. convexity), as the next example shows.

Example 3.4. For each positive integer $n$, let $I_{n}=\left[4^{-n}, 2.4^{-n}\right] \subset \boldsymbol{R}$. Let $A$ be the union of the $I_{n}$ 's together with 0 , so that $A$ is a closed admissible subset of $\boldsymbol{R}$. Define $f: A \rightarrow R$ by $f(x)=\left(4^{-n}\right)^{2}=4^{-2 n}$ if $x \in I_{n}$ and $f(0)=0$. Then $f$ is $C_{s}^{1}$ with $\mathrm{d} f(x)=0$ for all $x \in A$, and hence $f$ is $C_{s}^{\infty}$ differentiable. To see that $\mathrm{d} f(0)=0$, let $x \in I_{m} \cup\{0\}$ and $y \in I_{n}$ with $m>n$. Then $|y-x| \geqq \frac{1}{2} 4^{-n}$ and $|f(y)-f(x)| \leqq$ $\leqq f(y)=4^{-2 n} \leqq 2 \cdot 4^{-n}|y-x|$. Thus $\mathrm{d} f(0)=0$. Now if $f$ is $C_{w}^{2}$ in terms of $\left\{\mathrm{D} f, \mathrm{D}^{2} f\right\}$, then necessarily $\mathrm{D} f=\mathrm{d} f=0, \mathrm{D}^{2} f=\mathrm{d}^{2} f=0$, and by Whitney's theorem there is a $C^{2}$ map $g: \boldsymbol{R} \rightarrow \boldsymbol{R}$ such that $g\left|A=f, g^{\prime}\right| A \equiv 0$, and $g^{\prime \prime} \mid A \equiv 0$. An application of Taylor's theorem to $g$ with $x=4^{-(n+1)}$ and $y=4^{-n}$ yields a point $c_{n}$ between $x$ and $y$ such that

$$
g(y)=g(x)+g^{\prime}(x)(y-x)+\frac{1}{2} g^{\prime \prime}\left(c_{n}\right)(y-x)^{2} .
$$

But $g(y)=y^{2}, g(x)=x^{2}$, and $g^{\prime}(x)=0$ imply that

$$
g^{\prime \prime}\left(c_{n}\right)=2 \frac{y+x}{y-x}=\frac{10}{3} .
$$

This cannot happen since $g^{\prime \prime}$ is continuous and $g^{\prime \prime}(0)=0$. Hence $f$ does not have a $C^{2}$ extension to any neighborhood of 0 and $f$ is not $C_{w}^{2}$. This example also shows that the definition of $C_{w}^{k}$ differentiability is not inductive, since $f$ is $C_{w}^{1}$ in terms of $\{\mathrm{d} f\}$ and $\mathrm{d} f$ is $C_{w}^{1}$ in terms of $\left\{\mathrm{d}^{2} f\right\}$, but $f$ is not $C_{w}^{2}$ in terms of $\left\{\mathrm{d} f, d^{2} f\right\}$.

Theorem 3.5. Let $A \subset \boldsymbol{R}^{p}$ be a convex set, $f: A \rightarrow \boldsymbol{R}^{q}$ a map, and $k$ a positive integer. For each positive integer $n \leqq k$, let $\mathrm{D}^{n} f$ be a continuous map from $A$ into $L_{n}^{s}\left(\boldsymbol{R}^{p}, \boldsymbol{R}^{q}\right)$ such that $\mathrm{D}^{n} f(x)$ is a Fréchet derivative of $\mathrm{D}^{n-1} f$ at $x$ for each $x \in A$, where $\mathrm{D}^{0} f=f$. Then $f$ is $C_{w}^{k}$ in terms of $\left\{\mathrm{D}^{n} f\right\}_{n=1}^{k}$. If $A$ is also closed, then $f$ is $C^{k}$ differentiable.

Proof. Let $a \in A$ and let $\varepsilon>0$. Then there is a $\delta>0$ such that for each positive integer $n \leqq k,\left|\mathrm{D}^{n} f(y)-\mathrm{D}^{n} f(x)\right|<\varepsilon$ whenever $x, y \in B_{\delta}(a) \cap A$. Let $x, y \in B_{\delta}(a) \cap$ $\cap A$ and define $\alpha:[0,1] \rightarrow \boldsymbol{R}^{p}$ by $\alpha(t)=t x+(1-t) y$. Since $B_{\delta}(a) \cap A$ is convex, $\alpha(t) \in B_{\delta}(a) \cap A$ for each $t \in[0,1]$. Let $h=f \circ \alpha$. It follows then that $h$ is $C^{k}$ differentiable (in the ordinary sense with one-sided derivatives at 0 and 1) and that $h^{(n)}(t)=\mathrm{D}^{n} f(\alpha(t))(y-x)^{n}, n \leqq k$, where $\mathrm{D}^{n} f(\alpha(t))(y-x)^{n}$ is the number that results from placing $y-x$ in each of the $n$ arguments of $\mathrm{D}^{n} f(\alpha(t))$. Integrating by parts in the usual way leads to the equation

$$
h(1)=h(0)+h^{\prime}(0)+\ldots+\frac{1}{(k-1) !} h^{(k)}(0)+\frac{1}{(k-1) !} \int_{0}^{1}(1-t)^{k-1} h^{(k)}(t) \mathrm{d} t .
$$


Rewriting this equation in terms of $f, \alpha, y$, and $x$ yields the equation

Now

$$
\begin{aligned}
f(y)= & f(x)+\mathrm{D} f(x)(y-x)+\ldots+\frac{1}{(k-1) !} \mathrm{D}^{k-1} f(x)+ \\
& +\frac{1}{(k-1) !} \int_{0}^{1}(1-t)^{k-1} \mathrm{D}^{k} f(\alpha(t))(y-x)^{k} \mathrm{~d} t
\end{aligned}
$$

$$
\frac{1}{k !} \mathrm{D}^{k} f(x)(y-x)^{k}=\frac{1}{(k-1) !} \int_{0}^{1}(1-t)^{k-1} \mathrm{D}^{k} f(x)(y-x)^{k} \mathrm{~d} t
$$

and hence

$$
\begin{gathered}
\left|f(y)-\sum_{n=0}^{k} \frac{1}{n !} \mathrm{D}^{n} f(x)(y-x)^{n}\right|= \\
=\left|\frac{1}{(k-1) !} \int_{0}^{1}(1-t)^{k-1}\left[\mathrm{D}^{k} f(\alpha(t))-\mathrm{D}^{k} f(x)\right](y-x)^{k} \mathrm{~d} t\right| \leqq \frac{\varepsilon}{k !}|y-x|^{k} .
\end{gathered}
$$

In a similar way,

$$
\left|\mathrm{D}^{m} f(y)-\sum_{n=0}^{k-m} \frac{1}{n !} \mathrm{D}^{m+n} f(x)(y-x)^{n}\right| \leqq \frac{\varepsilon}{(k-m) !}|y-x|^{k-m}
$$

for each positive integer $m \leqq k$ and thus $f$ is $C_{w}^{k}$ in terms of $\left\{\mathrm{D}^{n} f\right\}_{n=1}^{k}$. If $A$ is also closed, then $f$ has a $C^{k}$ extension to $\boldsymbol{R}^{p}$ by Whitney's theorem and $f$ is $C^{k}$ differentiable. This completes the proof.

Corollary 3.6. Let $A \subset \boldsymbol{R}^{p}$ be closed, convex, and admissible, let $f: A \rightarrow \boldsymbol{R}^{q}$ be a map, and let $k$ be a positive integer or infinity. Suppose that for each positive integer $n \leqq k, \mathrm{D}^{n} f$ is a map from $A$ into $L_{n}\left(\boldsymbol{R}^{p}, \boldsymbol{R}^{q}\right)$. Then these are equivalent:

(1) For each $n \leqq k, \mathrm{D}^{n} f$ is continuous and $\mathrm{D}^{n} f(x)$ is a Fréchet derivative of $\mathrm{D}^{n-1} f$ at $x$ for each $x \in A$.

(2) The map $f$ is $C_{s}^{k}$ differentiable and $\mathrm{D}^{n} f(x)=\mathrm{d}^{n} f(x)$ for each $x \in A$ and each $n \leqq k$.

(3) The map $f$ is $C_{w}^{k}$ differentiable in terms of $\left\{\mathrm{D}^{n} f\right\}_{n=1}^{k}$.

(4) The map $f$ has a $C^{k}$ extension $g$ to $R^{p}$ and $g^{(n)}(x)=\mathrm{D}^{n} f(x)$ for each $x \in A$ and each $n \leqq k$.

Proof. Statements (3) and (4) are equivalent by Whitney's theorem. Now (3) implies (2) and (2) implies (1) whenever the domain $A$ is an admissible set. If (1) holds, then $\mathrm{D}^{n} f(x)$ is symmetric for $x \in A^{0}$ and by the continuity of $\mathrm{D}^{n} f, \mathrm{D}^{n} f(x)$ is symmetric for all $x \in A$. Hence (1) implies (3) by Theorem 3.5. This completes the proof.

Theorem 3.7. Let $C \subset R^{p}$ be closed, convex, and admissible. Let $U \subset \boldsymbol{R}^{p}$ be an 
open set, $A=U \cap C, f: A \rightarrow R^{q}$ a map, and $k$ a positive integer or infinity. Then these are equivalent:

(1) The map $f$ is $C_{s}^{k}$ differentiable.

(2) The map $f$ is $C^{k}$ differentiable.

Proof. The set $A$ is admissible by Lemma 2.2 and thus (2) implies (1). Suppose that $f$ is $C_{s}^{k}$ differentiable and let $a \in A$. Then there is a $\delta>0$ such that $B_{\delta}(a)^{*} \subset U$. Now $\left(B_{\delta}(a) \cap C\right)^{*}$ is closed, convex, admissible, and contained in $A$. By Corollary $3.5, f \mid\left(B_{\delta}(a) \cap C\right)^{*}$ has a $C^{k}$ extension $g$ to $\boldsymbol{R}^{p}$. Then $g \mid B_{\delta}(a)$ is a $C^{k}$ map which agrees with $f$ on $B_{\delta}(a) \cap A$. Hence $f$ is $C^{k}$ differentiable and the proof is complete.

4. Differentiable manifolds with generalized boundary. Let $\boldsymbol{E}$ be a Banach space. An $\boldsymbol{E}$-manifold with generalized boundary, or simply an $\boldsymbol{E}$-manifold, is a topological space $M$ such that for each $p \in M$ there is an open set $U$ about $p$ and a homeomorphism $\varphi$ from $U$ onto an admissible subset of $\boldsymbol{E}$.

An $E$-manifold is a $T_{1}$ space, but it need not be Hausdorff or paracompact. Now a regular $T_{1}$ space satisfying the second axiom of countability is metrizable and therefore paracompact (cf. Kelley [10], pp. 125, 160].) Since a paracompact space is a regular Hausdorff space, we have the result that an $\boldsymbol{E}$-manifold having a countable base for its topology is paracompact if and only if it is regular. However, an $\boldsymbol{E}$ manifold need not be a regular space. For example, let $M \subset R^{2}$ consist of the open upper half plane with its usual topology and the set of rational points on the $x$-axis, where a basic open-neighborhood of a rational point $x_{0}$ on the $x$-axis is the open upper half of a disc with center at $x_{0}$ together with $x_{0}$. Then $M$ is a Hausdorff $\boldsymbol{R}^{2}$ manifold with a countable base for its topology, but $M$ is not regular and hence not paracompact.

An $n$-manifold (with generalized boundary) is a regular $\boldsymbol{R}^{n}$-manifold satisfying the second axiom of countability. It follows from the comments in the preceeding paragraph that an $n$-manifold is Hausdorff and paracompact.

An atlas for an $\boldsymbol{E}$-manifold $M$ is a collection $\mathscr{A}$ of functions satisfying:

(i) each $\varphi \in \mathscr{A}$ is a homeomorphism from an open subset $\operatorname{dom} \varphi$ of $M$ onto an admissible subset im $\varphi$ of $\boldsymbol{E}$.

(ii) $M=\cup \operatorname{dom} \varphi(\varphi \in \mathscr{A})$.

If $\varphi$ and $\psi$ are members of the atlas $\mathscr{A}$, then the domain $\varphi(\operatorname{dom} \varphi \cap \operatorname{dom} \psi)$ of $\psi \circ \varphi^{-1}$ is an open subset of im $\varphi$ and therefore an admissible subset of $\boldsymbol{E}$ by Lemma 2.2. An atlas $\mathscr{A}$ for $M$ is a $C_{s}^{k}\left(C^{k}\right)$ atlas if $\psi \circ \varphi^{-1}$ is $C_{s}^{k}\left(C^{k}\right)$ differentiable for each $\varphi, \psi \in \mathscr{A}$.

Let $\mathscr{A}$ be a $C_{s}^{k}\left(C^{k}\right)$ atlas for the $\boldsymbol{E}$-manifold $M$ and let $\mathscr{D}$ be the collection of all homeomorphisms $\psi$ from an open subset of $M$ onto an admissible subset of $\boldsymbol{E}$ such that $\psi \circ \varphi^{-1}$ and $\varphi \circ \psi^{-1}$ are $C_{s}^{k}\left(C^{k}\right)$ differentiable for each $\varphi \in \mathscr{A}$. Then $\mathscr{D}$ is the unique $C_{s}^{k}\left(C^{k}\right)$ atlas for $M$ which contains $\mathscr{A}$ and is not properly contained in any $C_{s}^{k}\left(C^{k}\right)$ atlas for $M$. 
A $C_{s}^{k}\left(C^{k}\right)$ differentiable structure for the $\boldsymbol{E}$-manifold $M$ is a maximal $C_{s}^{k}\left(C^{k}\right)$ atlas for $M$. A $C_{s}^{k}\left(C^{k}\right)$ manifold is a pair $(M, \mathscr{D})$ where $M$ is an $\boldsymbol{E}$-manifold for some $\boldsymbol{E}$ and $\mathscr{D}$ is a $C_{s}^{k}\left(C^{k}\right)$ differentiable structure for $M$. If $\mathscr{A}$ is a $C_{s}^{k}\left(C^{k}\right)$ atlas for $M$, then members of the unique $C_{s}^{k}\left(C^{k}\right)$ differentiable structure containing $\mathscr{A}$ are called charts. A chart at $p$ is a chart containing $p$ in its domain.

Let $(M, \mathscr{D})$ be a $C_{s}^{k}\left(C^{k}\right)$ manifold. If $p$ is a point of $M$ such that for some chart $\varphi$ at $p, \varphi(p)$ is an interior point of $\operatorname{im} \varphi$, then $p$ is an interior point of im $\psi$ for each $\psi \in \mathscr{D}$. The boundary of $M$, denoted by $\partial M$, is the set of all points $p$ of $M$ such that for some (and hence every) chart $\varphi$ at $p, \varphi(p)$ is not an interior point of im $\varphi$. The manifold $M$ is a manifold without boundary if $\partial M=\emptyset$ or equivalently if for each chart $\varphi$ the image of $\varphi$ is open. Since $C^{k}$ and $C_{s}^{k}$ differentiability agree on open subsets of $\boldsymbol{E}$, the following theorem is immediate.

Theorem 4.1. Let $M$ be an $E$-manifold. If $\mathscr{A}$ is an atlas for $M$ such that for each $\varphi \in \mathscr{A}, \operatorname{im} \varphi$ is an open subset of $\boldsymbol{E}$, then $\mathscr{A}$ is a $C^{k}$ atlas if and only if $\mathscr{A}$ is a $C_{s}^{k}$ atlas. Hence $(M, \mathscr{D})$ is a $C^{k}$ manifold without boundary if and only if $(M, \mathscr{D})$ is a $C_{s}^{k}$ manifold without boundary.

A half-space in $\boldsymbol{E}$ is a set of the form $E_{\lambda}=\{x \in \boldsymbol{E}: \lambda(x) \geqq 0\}$ for some functional $\lambda \in L(E, R)$. A corner in $\boldsymbol{E}$ is a set of the form $\cap E_{\lambda}(\lambda \in \Lambda)$ for some linearly independent set $\Lambda \subset L(\boldsymbol{E}, \boldsymbol{R})$. The manifold $(M, \mathscr{D})$ is a manifold with smooth boundary (manifold with corners) if there is an atlas $\mathscr{A} \subset \mathscr{D}$ for $M$ such that im $\varphi$ is an open subset of a half-space (of a corner) in $\boldsymbol{E}$ for each $\varphi \in \mathscr{A}$.

Theorem 4.2. Let $M$ be an n-manifold. If $\mathscr{A}$ is an atlas for $M$ such that for each $\varphi \in \mathscr{A}, \operatorname{im} \varphi$ is an open subset of a half-space or a corner in $R^{n}$, then $\mathscr{A}$ is a $C^{k}$ atlas if and only if $\mathscr{A}$ is a $C_{s}^{k}$ atlas. Hence $(M, \mathscr{D})$ is a $C^{k}$ manifold with smooth boundary (with corners) if and only if $(M, \mathscr{D})$ is a $C_{s}^{k}$ manifold with smooth boundary (with corners.)

Proof. This theorem follows immediately from Theorem 3.7.

Let $M$ and $N$ be $C_{s}^{k}$ manifolds and let $f: M \rightarrow N$ be a map. Then $f$ is $C_{s}^{r}$ differentiable $(r \leqq k)$ if $f$ is continuous and for each chart $\varphi$ for $M$ and each chart $\psi$ for $N$, the composition $\psi \circ f \circ \varphi^{-1}$ is $C_{s}^{r}$ differentiable. The class of $C_{s}^{k}$ manifolds is a category with morphisms the $C_{s}^{k}$ differentiable functions. By Theorems 4.1, 4.2 and Theorem 3.7, this category includes $C^{k}$ manifolds without boundary and $C^{k}$ $n$-manifolds with smooth boundary or with corners. Thus we are able to give an intrinsic description of a convenient class of differentiable manifolds with boundary. In addition, one may extend the usual constructions of differential geometry (e.g. tangent spaces, the tangent bundle, etc.) to manifolds with generalized boundary in standard ways (cf. [5]).

5. Differentiable semigroups. In this section we apply the Parametrized Mapping Theorem (Theorem 2.11) to differentiable semigroups and their actions. 
A topological semigroup is a pair $(S, m)$ where $S$ is a Hausdorff space and $m: S \times$ $\times S \rightarrow S$ is a continuous function such that if $x, y, z \in S$, then $m(m(x, y), z)=$ $=m(x, m(y, z))$. Throughout this section $(S, m)$ is contracted to $S$, and $m(x, y)$ is denoted by $x y$. Semigroup means topological semigroup. A monoid is a semigroup with a two-sided identity element, usually denoted by 1 .

A $C_{s}^{k}$ semigroup $(k \geqq 1)$ is a semigroup $S$ on a $C_{s}^{k}$ manifold (with generalized boundary) such that the multiplication $m: S \times S \rightarrow S$ is $C_{s}^{k}$ differentiable. Examples of $C_{s}^{k}$ semigroups include Lie groups, the semigroup $M_{n}(\boldsymbol{R})$ of all $n \times n$ real matrices, the Lie monoids of Hille and Philips [6], cones in $\boldsymbol{R}^{n}$, and ray semigroups. Precisely, let $G$ be a Lie group with Lie algebra $L(G)$ and let $D \subset L(G)$. The ray semigroup generated by $D$ is the subsemigroup of $G$ generated by the set of elements of the form $\exp (t X)$, where $t \geqq 0$ and $X \in D$. Thus, a cone is a commutative ray semigroup. Jurdjevic and Sussmann have shown [9] that a ray semigroup $S$ has dense interior in the Lie subgroup it generates. It follows that $S$ is a $C_{s}^{\infty}$ monoid, although the identity of $S$ may lie at an irregular point of the boundary (e.g. a corner or a cusp). Ray semigroups arise in the control theory of certain nonlinear systems on manifolds (cf. Brockett [2], Hirschorn [8], Jurdjevic and Sussmann [9]). Other examples of $C_{s}^{\infty}$ semigroups are subsemigroups of $M_{n}(\boldsymbol{R})$ which have as underlying space an admissible subset of a submanifold of $M_{n}(\boldsymbol{R})$. For instance the semigroup

$$
S=\left\{\left(\begin{array}{ll}
x & y \\
0 & 1
\end{array}\right): x, y \geqq 0, x+y \leqq 1\right\}
$$

is a $C_{s}^{\infty}$ monoid.

Let $S$ be a monoid and let $M$ be a space. An action of $S$ on $M$ is a continuous map $\pi: S \times M \rightarrow M$ such that: (i) $\pi(1, p)=p$, for all $p \in M$, and (ii) $\pi(x y, p)=$ $=\pi(x, \pi(y, p))$, for all $x, y \in S$, and all $p \in M$. When no confusion seems possible, $\pi(x, p)$ is contracted to $x p$. For each $x \in S, \pi_{x}: M \rightarrow M$ is the map defined by $\pi_{x}(p)=x p$.

Theorem 5.1. Let $S$ be a monoid on a $C_{s}^{k}$ manifold and let $M$ be a $C_{s}^{k}$ manifold. Let $\pi$ be an action of $S$ on $M$ such that $\pi$ is strongly differentiable at $(1, p)$, for some $p \in M$. Then there are open sets $U \subset S$ about 1 and $V \subset M$ about $p$ such that if $x \in U$, then $\pi_{x} \mid U$ is a homeomorphism onto an admissible subset of $M$.

Proof. By using charts at 1 and $p$, we may assume that $S$ is an admissible subset of $\boldsymbol{E}$ and $M$ is an admissible subset of $\boldsymbol{F}$, where $\boldsymbol{E}$ and $\boldsymbol{F}$ are Banach spaces. Since $\pi_{1}: M \rightarrow M$ is the identity map, $\mathrm{d}_{2} \pi(1, p): \boldsymbol{F} \rightarrow \boldsymbol{F}$ is the identity map. By Theorem 2.11 , there are open sets $U$ and $V$ as required and the proof is complete.

Corollary 5.2 (Local Cancellation). Let $S$ be a monoid on a $C_{s}^{k}$ manifold such that $m: S \times S \rightarrow S$ is strongly differentiable at $(1,1)$. Then there is an open set $W$ about 1 such that if $x, y, z \in W$ and $x y=x z$, then $y=z$. 
Proof. The multiplication $m$ is an action of $S$ on itself. By Theorem 5.1, there are open sets $U$ and $V$ about 1 such that if $x \in U$ and $y, z \in V$ with $x y=x z$, then $y=z$. Let $W=U \cap V$ and the proof is complete.

A $C_{s}^{\infty}$ monoid is not necessarily embeddable in a group (e.g. $\left.M_{n}(\boldsymbol{R})\right)$. However, it is shown in [4] that a $C_{s}^{\infty}$ monoid is locally (near 1) embeddable in a Lie group. Our final application of Theorem 2.11 concerns the group of units of a monoid.

Let $S$ be a monoid. The group of units of $S$, denoted by $H(1)$, is the set of elements of $S$ having a two-sided inverse. Clearly, $H(1)$ is a group.

Theorem 5.3. Let $S$ be a $C_{s}^{k}$ monoid. Suppose that 1 is not a boundary point of $S$. Then $H(1)$ is an open subset of $S$.

Proof. By using a chart at 1 , we may assume that $S$ is an admissible subset of the Banach space $\boldsymbol{E}$ and that $1 \in S^{0}$. Let $U \subset S^{0}$ be an open set about 1 such that if $x, y, z \in U$ and $x y=x z$, then $y=z$. Let $V$ be an open set about 1 such that $V . V \subset$ $\subset U$. By Theorem 2.11, there is an open set $N$ about 1 such that $1 \in x V$ for each $x \in N$. Let $x \in N \cap V$. Then there is a $y \in V$ with $x y=1$. Now $y x \in V^{2} \subset U$ and $y x y x=y x$. By cancellation in $U, y x=1$. Thus, $N \cap V \subset H(1)$. Since translation by an element of $H(1)$ is a homeomorphism of $S, H(1)$ is open and the proof is complete.

Re mark. Mostert and Shields showed in [13] that if $S$ is finite-dimensional, then continuity of the multiplication is sufficient to prove that $H(1)$ is open. Moreover, $H(1)$ is a Lie group in this case.

\section{References}

[1] Banach S.: Théorie des Opérations Linéaires, Warsaw, 1932.

[2] Brockett R. W.: Lie algebras and Lie groups in control theory, Geometric Methods in System Theory, Reidel, Boston (1973), 43-82.

[3] Dieudonné J.: Foundations of Modern Analysis, Academic Press, New York and London (1960).

[4] Graham G.: Manifolds with Generalized Boundary and Differentiable Semigroups, Ph.D. Thesis, University of Houston, 1979.

[5] Graham G.: The Lie theory of differentiable semigroups (to appear).

[6] Graves L. M.: Some mapping theorems, Duke Math. J. 17 (1950), 111-114.

[7] Hille E. and Phillips R. S.: Functional Analysis and Semigroups, Am. Math. Soc., Providence (1957).

[8] Hirschorn R.: Topological semigroups, sets of generators and controllability; Duke Math. J., 40 (1973), 937-- 947.

[9] Jurdjevic V. and Sussmann H. J.: Control systems on Lie groups, Jour. Differential Eq. 12 (1972), 313-329.

[10] Kelley J. L.: General Topology, Graduate Texts in Mathematics, vol. 27, Springer-Verlag, New York, Heidelberg, and Berlin.

[11] Lang S.: Introduction to differentiable manifolds, Interscience, New York (1967). 
[12] Leach E. B.: A note on inverse function theorems, Proc. Amer. Math. Soc. 12 (1961), 694 to 697.

[13] Mostert P. S. and Shields A. L.: Semigroups with identity on a manifold, Trans. Am. Math. Soc., 91 (1959), 380-- 389.

[14] Nashed M. Z.: Differentiability and related properties of nonlinear operators: Some aspects of the role of differentials in nonlinear functional analysis. Nonlinear Functional Analysis and Applications, L. B. Rall, ed., Academic Press, New York (1971), 109-309.

[15] Nashed M. Z.: Generalized inverse mapping theorems and related applications of generalized inverses in nonlinear analysis, Nonlinear Equations in Abstract Spaces, V. Lakshmikantham, ed., Academic Press, New York (1978), 217-252.

[16] Nijenhuis A.: Strong derivatives and inverse mappings, Amer. Math. Monthly 81 (1974), 969-981.

[17] Vainberg M. M.: Variational Methods for the Study of Nonlinear Operators, Holden-Day, San Francisco, (1964).

[18] Whitney $H$.: Analytic extensions of differentiable functions defined in closed sets, Trans. Amer. Math. Soc. 36 (1934), 63-89.

Author's address: Texas A\&M University, College Station, Texas 77843, USA. 